Revue d'histoire de l'enfance " irrégulière »

Le Temps de l'histoire

16 | 2014

La part scolaire : jeunesse irrégulière et école $\left(\mathrm{XIX}^{\mathrm{e}}\right.$ $\mathrm{XX}$ siècles)

\title{
Dominique Dessertine, Enfance, santé et société
}

Jean-Jacques Yvorel

\section{(2) OpenEdition}

1 Journals

Édition électronique

URL : https://journals.openedition.org/rhei/3703

DOI : 10.4000/rhei.3703

ISBN : 978-2-7535-4019-4

ISSN : $1777-540 \mathrm{X}$

Éditeur

Presses universitaires de Rennes

Édition imprimée

Date de publication : 30 octobre 2014

Pagination : 209-212

ISBN : 978-2-7535-3558-9

ISSN : $1287-2431$

Référence électronique

Jean-Jacques Yvorel, « Dominique Dessertine, Enfance, santé et société », Revue d'histoire de l'enfance " irrégulière » [En ligne], 16 | 2014, mis en ligne le 30 octobre 2014, consulté le 09 septembre 2021. URL : http://journals.openedition.org/rhei/3703 ; DOI : https://doi.org/10.4000/rhei.3703

Ce document a été généré automatiquement le 9 septembre 2021.

(c) PUR 


\title{
Dominique Dessertine, Enfance, santé et société
}

\author{
Jean-Jacques Yvorel
}

\section{RÉFÉRENCE}

Dominique Dessertine, Enfance, santé et société, Lyon, LARHRA-RESEA, 2013, Chrétiens et sociétés, Documents et Mémoires, n¹8, 375 p., ISBN : 979-10-91592-00-0

Il peut sembler délicat de recenser dans la Revue d'histoire de l'enfance "irrégulière" l'ouvrage de Dominique Dessertine alors que son préfacier, Olivier Faure, la présente à juste titre comme l'une des chevilles ouvrières de ladite revue. Si l'on ajoute à ce fait l'immense estime de l'auteur de ces lignes pour une historienne aussi modeste que rigoureuse, la neutralité axiologique et l'objectivité scientifique de ce compte rendu critique peuvent être mises en doute. Mais comment ne pas rendre compte dans notre revue des travaux de l'une des pionnières d'un champ historiographique qui est son objet même? Il ne restera au lecteur qui lirait dans mon propos la marque d'une complaisance coupable, l'expression d'une opinion déformée par l'amitié qu'à agir en historien, c'est-à-dire à retourner aux sources et à lire le livre de Dominique Dessertine.

2 L'ouvrage rassemble vingt-deux articles qui ont été regroupés en trois rubriques: enfance (11 articles), santé ( 6 articles), société ( 5 articles). L'enfance et la jeunesse constituent donc un thème particulièrement travaillé par la chercheuse du Laboratoire de recherche historique Rhône-Alpes (LARHRA). Si l'auteur de ces lignes connaissait la plupart des textes ici rassemblés pour les avoir lus lors de leur parution initiale, leur lecture en continu permet de mieux mettre à jour les caractéristiques de la démarche historienne de Dominique Dessertine. Trois mots permettent de la caractériser: novation, cohérence et rigueur.

3 Novateurs les travaux de Dominique Dessertine le sont par les objets qu'elle choisit tant en matière d'histoire de la santé qu'en matière d'histoire de l'enfance et de la jeunesse. Pour s'en tenir à ce dernier champ qui nous intéresse plus particulièrement, après un 
petit détour par les volumes de la Bibliographie annuelle d'histoire de France afin d'établir la chronologie des faits, on constate le caractère pionnier des articles sur la loi de 1889, sur les ancêtres des centres de loisirs que sont les patronages - cosignés avec son époux Bernard Maradan (1946-2000) -, sur les tribunaux pour enfants et adolescents de la loi de 1912, sur les écoles de plein-air de l'entre-deux-guerres ou sur les centres sociaux. Autre exemple, si aujourd'hui faire la sociogenèse des concepts et des catégories qui servent à décrire le monde social et à classer les populations semble " naturel », quand la chercheuse lyonnaise rédige sa contribution sur « la catégorisation de l'enfance par la justice» il n'en est rien. Pierre Bourdieu n'a pas encore publié son entretien avec Lutz Raphael ${ }^{1}$ ni Gérard Noiriel ses réflexions sur la socio-histoire ${ }^{2}$ et l'usage irréfléchi des catégories juridico-administratives est plus souvent la règle que l'exception.

Chez Dominique Dessertine, la créativité ne se limite pas aux choix de sujets neufs. La démarche aussi est innovante. Lisons attentivement pour commencer l'article qui ouvre ce recueil - "L'Émergence de la politique sociale de l'enfance: des enfants trouvés à l'enfance assistée (1780-1940)» - en ne perdant pas de vue la date de sa publication: 1989. L'auteure y analyse de façon "grossière, rapide et schématisée à l'extrême ", selon les propres dires (p. 29), l'évolution des prises en charge sociales de l'enfance. Elle tisse des liens entre assistance et répression, entre, par exemple, la loi civile de 1889 sur les enfants moralement abandonnés et la loi pénale de 1912 sur les tribunaux pour enfants et adolescents et la liberté surveillée. Or, l'article est rédigé à une époque où l'auteur d'une monographie sur une colonie agricole pénitentiaire pouvait ne pas voir dans les effectifs de cette dernière les pupilles de l'assistance qui s'y trouvaient avant même le vote de la loi du 28 juin 1904 relative à l'éducation des pupilles de l'Assistance publique difficiles et vicieux ${ }^{3}$. Dominique Dessertine nous invite donc à prendre en compte toutes les formes "d'irrégularité juvénile» et toutes les institutions qui interviennent dans ce secteur. C'est exactement le projet de notre revue... mais son premier numéro date de 1998.

5 La nouveauté en histoire passe bien souvent par l'exploitation d'archives négligées ou de sources jusque-là méprisées. Là encore Dominique Dessertine est particulièrement performante. L'exemple le plus éclatant de cette « imagination archivistique » est peutêtre l'usage qu'elle fait avec Bernard Maradan dans son travail sur les patronages, des bulletins paroissiaux, « une source [nous dit-elle], largement méconnue des historiens » (p. 170).

6 Enfin dans chaque article, Dominique Dessertine ne manque pas d'ouvrir des pistes de recherche que bien souvent elle exploite elle-même par la suite comme lorsqu'elle évoque «la médicalisation des institutions d'assistance/répression de l'enfance ${ }^{4} »$ (p. 29).

7 La cohérence de Dominique Dessertine n'est pas à rechercher dans l'utilisation privilégiée d'une méthode. En la matière, elle navigue de l'histoire quantitative à la micro-histoire, de l'analyse des textes normatifs au recueil de témoignages oraux, « du dépouillement sériel des dossiers judiciaires à la lecture des thèses et articles de médecine ». C'est dans la démarche même, dans la volonté de toujours articuler son travail autour de deux axes fondamentaux que réside, nous semble-t-il, la grande cohérence de l'œuvre de Dominique Dessertine. D'abord, quel que soit l'objet empirique qu'elle étudie, elle va systématiquement s'efforcer de comparer le prescrit, les discours, les programmes et les pratiques effectives et focaliser son analyse sur les raisons et la signification des décalages qu'elle a mis à jour. Ensuite, même si elle se penche sur 
l'histoire d'une "petite» association locale, elle va la relier au contexte démographique, économique, social, cognitif/intellectuel. Aussi, alors même que Dominique Dessertine a plus fréquenté les archives départementales, municipales, diocésaines de la région lyonnaise que les archives nationales, elle échappe totalement au travers de l'érudition locale.

Ce recueil nous propose trois inédits. Il s'agit en fait de communications prononcées à l'occasion de manifestations commémoratives qui n'ont pas donné lieu à des publications. La première est consacrée au «Cent cinquante ans de l'orphelinat SaintJoseph de Vienne » (p.111-127). La seconde au Prado, une autre œuvre pour enfants rhodanienne, fêtant elle aussi ses 150 ans $^{5}$. La troisième enfin ne concerne pas l'enfance ou la jeunesse puisqu'elle évoque les «Cinquante ans d'histoire de l'association des Veuves civiles de l'Isère » (p.301-315). Ces trois communications montrent toute la rigueur de Dominique Dessertine. Les commémorations peuvent donner lieu à des discours hagiographiques, à l'élaboration de mythes fondateurs, à l'évocation d'un âge d'or qui n'a jamais existé. Tout en faisant preuve d'un grand respect et d'une véritable empathie envers les militants associatifs qui l'ont invitée, Dominique Dessertine ne se déprend jamais des principes qui guident son travail d'historienne et continue d'interroger discours et pratiques tout en resituant la "petite» histoire de ces associations dans l'histoire sociale et générale.

C'est donc tout naturellement que le beau et riche ouvrage de Dominique Dessertine rejoindra la bibliothèque des lecteurs de notre revue où il viendra prendre place à côté de ceux qu'elle a déjà publiés dans le domaine socio-éducatif sur La société lyonnaise pour le sauvetage de l'enfance (érès, 1991), sur L'âge d'or des patronages (Vaucresson, 2001), ou sur Les centres sociaux (1880-1980) (Presses universitaires du Septentrion, 2004).

\section{NOTES}

1. BOURDIEU Pierre, «Sur les rapports entre la sociologie et l'histoire en Allemagne et en France, entretien avec Lutz Raphaël », Actes de la recherche en sciences sociales, $n^{\circ} 106-107$, mars 1995, p. 108-122.

2. NoIRIEL Gérard, Introduction à la socio-histoire, Paris, La découverte, 2006, $121 \mathrm{p}$.

3. Éric Pierre a montré la diversité des mineurs placés dans la colonie de Mettray dans Pierre (Éric), F.-A. Demetz et la colonie agricole de Mettray. Entre réformisme « romantique » et injonctions administratives, Paedagogica historica, 2002, vol. 38, n²-3, p. 451-466.

4. Dominique Dessertine a notamment dirigé le $n^{\circ} 6$ de notre revue consacré aux Sciences du psychisme et enfance "irrégulière ».

5. « Le Prado en son temps. Identité et adaptation », p. 149-163. 